\title{
475355 - PULSATILE CARDIO-PULMONARY BYPASS DOES NOT IMPROVE MICROVASCULAR FLOW: A PRELIMINARY REPORT
}

\author{
David Bracco, MD $^{1}$, Roy Kazan, none ${ }^{1}$, Marie-Claude Bluteau, BEng ${ }^{1}$, Dominique \\ Shum Tim, $\mathbf{M D}^{2}$, Christo Tchervenkov, $\mathbf{M D}^{2}$, Thomas Hemmerling, $\mathbf{M D}^{1}$ \\ 1. Anesthesiology, McGill, Montreal, QC, Canada \\ 2. Cardiac Surgery, McGill University, Montreal, QC, Canada
}

Introduction: Cardiac surgery under cardiopulmonary bypass induces numerous physiopathological changes and end organ damages. Interaction of blood with foreign membranes, hypothermia, non-pulsatile flow, micro bubbles and micro clots have been proposed in the physiopathology of these end organ damages. Pulsatile cardio-pulmonary bypass mimics part of the physiologic 'pulsatility' and has been advocated to decrease the degree of end organ damage. The aim of the present work was to assess the effect of pulsatile and non pulsatile cardiopulmonary bypass on in vivo micro-circulation. Methods: Local REB approval was obtained for this study. Twenty patients undergoing CABG surgery under CPB were enrolled. Microcirculation was assessed using an orthogonal polarisation spectroscope (OPS) under the tongue at 3 definite time points: before CPB, at the end of 20 min of pulsatile or 20 min non-pulsatile CPB. Pulsatile bypass was provided with the internal function of the pump at a rate of 60 /minute a baseline flow of 30\% and 1/1 systole/diastole ratio. The same pump flow was used in both phases. OPS allows to determine the functional capillary density, the flow characteristics of small capillaries. Data presented as median (25th, 75th quartile). Results: Preliminary 10 patients are reported. Their median age is 68.5 (range 57 - 85). Pre bypass cardiac output 3.45 (2.30 - 5.10) did not differ from pump flow 4.05 (3.05 5.10). Hematocrit dropped from $0.36(0.23-0.44)$ pre bypass to $0.23(0.19-0.31)$ during non pulsatile and .24 (.19 - .30) during pulsatile flow (NS). Arterial lactate was 1.0 (0.7 2.0) pre-bypass and 1.25 (0.7 - 2.0) during non pulsatile and 1.20 (0.7 - 1.9) during pulsatile CPB (NS). Non-pulsatile CPB increased the number of not and intermittently perfused capillaries and decreased the number of hyperdynamic vessels. Pulsatile CPB tends to be associated with more hyperdynamic capillaries $\mathrm{P}=0.08$ ) and less continuously perfused capillaries $(\mathrm{P}=0.05)$. (Figure) $\mathrm{CPB}$ is associated with a decrease in the functional capillary density and pulsatile CPB tends to blunt this effect with a greater capillary density, in particular for the single RBC capillaries (8-16 $\mu \mathrm{m})$.

Discussion: Cardio-pulmonary bypass using the same systemic flow and perfusion pressure than native cardiac output is associated with a decrease in functional capillary density. Pulsatile CPB tend to improve the microcirculatory effects of CPB. Further studies are required to better delineate this effect and assess the relationship between peri-operative microcirculatory disturbances and post-operative course.

References: This work was supported by the 2006 David S Sheridan Canadian Research Award. 

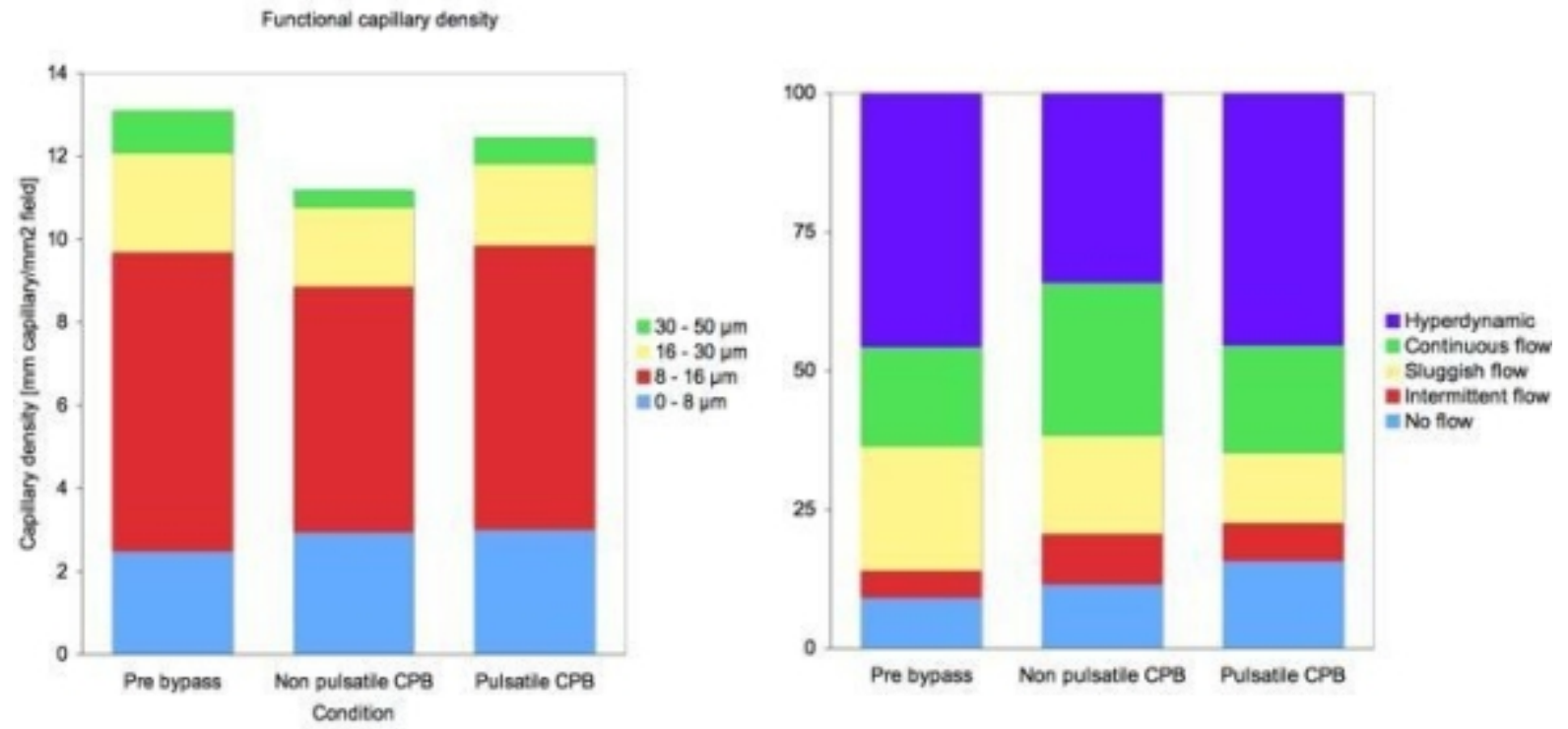